Apidologie, 1973, 4 (1), 65-80.

\title{
ERFAHRUNGEN MIT EINER NEUEN KINETISCHEN METHODE ZUR BESTIMMUNG DER DIASTASEZAHL IN HONIG UND ÜBER EIGENSCHAFTEN DER HONIG-DIASTASE
}

\author{
Expériences réalisées avec une nouvelle méthode cinétique \\ pour la détermination de l'indice de diastase du miel \\ et pour l'êtude des propriêtés de cette diastase
}

\author{
H. HADORN und K. ZÜRCHER
}

Zentrallaboratorium der Coop Schweiz, Basel

\section{SUMMARY}

\author{
EXPERIENCES WITH A NEW METHOD OF DETERMINING THE DIASTASE \\ NUMBER IN HONEY AND ON PROPERTIES OF HONEY DIASTASE
}

A recently by the authors elaborated method for determining the diastase number in honey was tested on various authentic honeys. The principle of the new kinetic method is as fallows : at $40^{\circ} \mathrm{C}$ a buffered original honey solution is acting upon a starch solution. At desired intervals samples of this reaction mixture are withdrawn and mixed with an iodine solution. The breakdown of the starch is observed photometrically. In a diagram the extinction is plotted against the corresponding reaction time. The diastatic activity is calculated from the initially linear reaction curve.

Beside the diastase number of the honey, the sucrase number, the water and the hydroxymethylfurfural content as well as the $\mathrm{pH}$ value and the acidity are determined. Some of the examined honeys were especially rich in enzymes whereas others were poor. Lavendine honeys very often are strikingly poor in enzymes. An inhibitory effect or even a substance poisonous to enzymes could not be detected in Lavendine honeys. Probably the natural poverty in enzymes of Lavendine honeys is caused by mass flows.

For the characterization of the honey diastase the pH optimum was determined (pH $=5,4)$; the activation of the diastase was stated by natrium chlorid. This statement points to an animal origin of the honey diastase. Honey dew honeys and floral honeys have diastases of the same properties. 


\section{ZUSAMMENFASSUNG}

Eine kürzlich von uns ausgearbeitete Methode zur Bestimmung der Diastasezahl in Honig wurde an verschiedenen authentischen Honigen ausprobiert. Das Prinzip der neuen kinetischen Methode ist folgendes : Eine gepufferte Honig-Stammlösung lässt man bei $40^{\circ} \mathrm{C}$ auf Stärkelösung einwirken. In bestimmten Zeitabständen entnimmt man diesem Reaktionsgemisch Proben und versetzt sie mit Jodlösung. Der Stärkeabbau wird photometrisch verfolgt. In einem Diagramm trägt man die Extinktion gegen die entsprechende Reaktionszeit auf. Aus der am Anfang linear verlaufenden Reaktionskurve wird die Diastaseaktivität berechnet.

Neben der Diastasezahl wurden in den Honigen die Saccharasezahl, der Wasser - und Hydroxymethylfurfurol - Gehalt sowie der pH-Wert und die Säure bestimmt. Unter den untersuchten Honigen befanden sich besonders enzymreiche, aber auch enzymarme Honige. Lavandin-Honige fallen oft durch ihre Armut an Enzymen auf. Eine enzymhemmende Wirkung oder gar eine Substanz, welche als Enzymgift wirkt, konnte in den Lavandin-Honigen nicht nachgewiesen werden. Die natürliche Enzymarmut gewisser Lavandin-Honige dürfte auf Massentracht zurückzuführen sein. Zur Charakterisierung der Honig-Diastase wurde das pH-Optimum bestimmt $(\mathrm{pH}=5,4)$ und die Aktivierung der Diastase durch Natriumchlorid nachgewiesen. Dieser Befund deutet auf die tierische Herkunft der Honig-Diastase hin. Honigtauhonige und Blütenhonige enthalten Diastase mit gleichen Eigenschaften.

\section{EINLEITUNG}

Die Diastaseaktivität (Amylaseaktivität) des Honigs wird seit Jahrzehnten benutzt, um zu prüfen, ob ein Honig naturbelassen und nicht durch übermässig starkes Erhitzen geschädigt worden ist. In der Literatur findet man bereits recht zahlreiche Methoden zur Bestimmung der Honigdiastase. Alle diese Methoden beruhen auf dem enzymatischen Abbau von Stärkelösung unter definierten, jedoch von einem Autor zum andern wechselnden Versuchsbedingungen. Die Methoden sind zum Teil recht umständlich, und die nach verschiedenen Verfahren gefundenen Enzymaktivitäten lassen sich nur bedingt miteinander vergleichen. Eine der bekanntesten Methoden zur Bestimmung der Honigdiastase stammt von Gothe (1914). Diese Methode ist von Kiermeier und KöвERLEIN (1954) modifiziert und verbessert worden. Eine brauchbare, photometrische Methode zur Bestimmung der Honigdiastase haben Schade, Marsh und Eckert (1958) beschrieben. Diese etwas umständliche Methode wurde von White und Pirent (1959), später auch von uns modifiziert (HADoRN 1961). Eine Variante dieser Methode ist in das Schweiz. Lebensmittelbuch (1967) aufgenommen worden. Die Methode liefert zuverlässige und gut reproduzierbare Werte. Die Arbeitsweise ist jedoch recht umständlich, erfordert viele Vorbereitungen und ist in Laboratorien, die nur gelegentlich einzelne Honige zu prüfen haben, wenig beliebt. Aus diesem Grunde haben wir (Zürcher und Hador 1972) kürzlich eine einfachere kinetische Methode zur Bestimmung der Honigdiastase ausgearbeitet. Dabei wird der Stärkeabbau in Abhängigkeit der Reaktionszeit photometrisch verfolgt (Jodstärke-Reaktion) und hieraus die Diastasezahl berechnet. Hier soll über Erfahrungen mit dieser Methode berichtet werden. 


\section{UNTERSUCHUNGSMATERIAL}

Eine Anzahl authentischer Schweizer Honige wurden uns in freundlicher Weise von Frl. Dr. A. Maurizio, Liebefeld Bern, zur Verfügung gestellt. Die Pollenanalyse sämtlicher Honigproben besorgte ebenfalls Frl. Dr. Maurizio. Von Herrn Dr. Louveaux, Station de Recherches sur l'Abeille et les Insectes sociaux, Bures-sur-Yvette (S.-et-O.) France, erhielten wir eine Anzahl Honige, vorwiegend aus Südfrankreich.

\section{UNTERSUCHUNGSMETHODEN}

Zur Untersuchung der Honige haben wir ausschliesslich Methoden benützt, welche in unserem Laboratorium überprüft oder speziell ausgearbeitet worden waren (HADoRN 1961, HadorN und Zürcher 1963, 1966). Die meisten dieser Methoden befinden sich im Schweiz. Lebensmittelbuch (1967).

\section{DIASTASEZAHL}

Die Diastasezahl bestimmten wir nach einer neuen kinetischen Methode. Eine ausführliche Arbeit hierüber ist an anderer Stelle erschienen (ZürCHER und HADORN 1972). Hier soll nur die Arbeitsvorschrift kurz wiedergegeben werden.

\section{Prinzip}

Eine gepufferte Honig-Stammlösung läßt man bei $40^{\circ} \mathrm{C}$ auf Stärkelösung einwirken. In bestimmten Zeitabständen entnimmt man diesem Reaktionsgemisch Proben und versetzt sie mit Jodlösung. Der Stärkeabbau wird photometrisch verfolgt. In einem Diagramm trägt man die Extinktion gegen die entsprechende Reaktionszeit auf. Aus der am Anfang linear verlaufenden Reaktionskurve wird die Diastaseaktivität berechnet.

\section{Definition}

Die Einheit der Diastaseaktivität ist jene Enzymmenge, die in einer Stunde unter den Versuchsbedingungen $10 \mathrm{mg}$ Stärke abbaut. Die Resultate werden in Diastase-Einheiten pro Gramm Honig angegeben. Die Diastasezahl gibt an, wieviele Gramm Stärke von den in $100 \mathrm{~g}$ Honig enthaltenen Enzymen in einer Stunde abgebaut werden.

\section{Geräte}

Reaktionskölbchen, siehe Abb. 1.

Das gut gereinigte Reaktionskölbchen sowie alle Glasgefässe, mit denen der Honig vor und während dem Enzymversuch in Berührung kommt, sind auszudämpfen und anschliessend im Trockenschrank zu trocknen.

Thermostatenbad von $40,0^{\circ} \mathrm{C}$.

\section{Reagenzien}

Natriumchloridlösung $4 \%$

$4,0 \mathrm{~g}$ Natriumchlorid werden mit frisch ausgekochtem destillierten Wasser gelöst und im Messkölbchen auf $100 \mathrm{ml}$ verdünnt.

\section{Stärkelösung}

Es sind nicht alle löslichen Stärkepräparate des Handels brauchbar, da die AnfangsExtinktion zwischen 0,5 und 0,6 liegen sollte (siehe Berechnung).

Von der löslichen Stärke (Noredux-Standardstärke, Siegfried AG Zo fingen, oder lösliche Stärke Merck) muss zunächst die Trockensubstanz bestimmt werden. Etwa $2 \mathrm{~g}$ Stärke werden auf dem Boden eines flachen Wägeglases (Durchmesser $5 \mathrm{~cm}$ ) in dünner Schicht ausgebreitet, dann gewogen und während $11 / 2$ Stunden bei $130^{\circ} \mathrm{C}$ getrocknet. Nach dem Abkühlen im Exsikkator wird zurückgewogen. Der Gewichtsverlust entspricht dem Wassergehalt.

Man berechnet die Menge lufttrockene Stärke, welche eingewogen werden muss, damit in 
der Einwaage (E) genau 0,500 g wasserfreie Stärke enthalten sind, nach folgender Formel : $\mathbf{E}=\frac{\mathbf{5 0}}{\mathbf{T}}$ wobei $\mathbf{T}=$ Trockensubstanz in $\%$.

- Die entsprechende Menge Stärke, welche $0,500 \mathrm{~g}$ wasserfreier Stärke entspricht auf der Analysenwaage abwägen.

- $20 \mathrm{ml}$ Wasser zusetzen und Stärke zu dünnem Brei anrühren.

- $5 \mathrm{ml}$ Puffer, $\mathrm{pH}=5,4$ zugeben und mit ca.

- $50 \mathrm{ml}$ kochendem Wasser übergiessen. Unter Rühren zum Sieden erhitzen. Abkühlen und mit Wasser auf $100 \mathrm{ml}$ verdünnen.

Substrat-Pufferlösung $\mathrm{pH}=5,4$.

$40,0 \mathrm{~g}$ di-Natriumhydrogenphosphat krist. $\mathrm{Na}_{2} \mathrm{HPO}_{2} \cdot 12 \mathrm{H}_{2} \mathrm{O}$ und

$9,29 \mathrm{~g}$ Citronensäure Monohydrat abwägen, in Wasser lösen und auf 1 Liter verdünnen.

Sistier-Pufferlösung $\mathrm{pH}=7,0$.

$58,9 \mathrm{~g}$ di-Natriumhydrogenphosphat krist. $\mathrm{Na}_{2} \mathrm{HPO}_{4} \cdot 12 \mathrm{H}_{2} \mathrm{O}$ und

$3,70 \mathrm{~g}$ Citronensäure Monohydrat abwägen, in Wasser lösen und auf 1 Liter verdünnen.

Jodlösung 0,002-n (frisch zubereitet)

$5,0 \mathrm{ml} \quad 0,1-\mathrm{n}$ Jodlösung mit

$10,0 \mathrm{~g}$ Kaliumjodid und Wasser auf $250 \mathrm{ml}$ stellen.

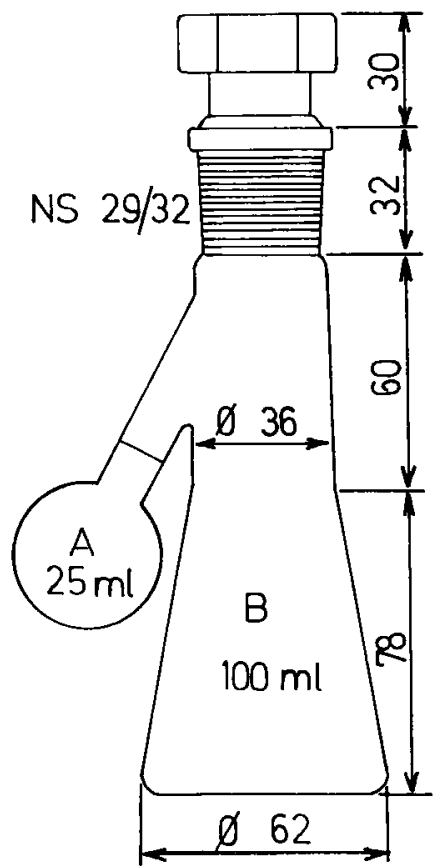

Aвв. 1. - Reaktionsgefäss für Diastasezahl-und Saccharasezahl-Bestimmung

FIG. 1. - Réacteur utilisé pour la détermination des indices de diastase et de saccharase

\section{Ausführung der Bestimmung}

a) Honiglösung $2 \%$

$1,00 \mathrm{~g}$ Honig in 50-ml-Becherglas auf $\pm 0,01 \mathrm{~g}$ genau abwägen, ca.

$25,00 \mathrm{ml}$ Substrat-Pufferlösung $\mathrm{pH}=5, \overline{4}$ zugeben und Honig lösen.

$1,50 \mathrm{ml}$ Natriumchloridlösung zusetzen und mit Pufferlösung $\mathrm{pH}=5,4$ im Messkölbchen auf $50 \mathrm{ml}$ verdünnen. 
b) Enzymversuch

- $20 \mathrm{ml}$ der frisch bereiteten $2 \%$-igen Honiglösung (entspricht $400 \mathrm{mg}$ Honig) in seitlichen Ansatz des Reaktionsgefässes pipettieren.

- $20 \mathrm{ml}$ Stärkelösung 0,5\% (entspricht $100 \mathrm{mg}$ Stärke) in das Kölbchen pipettieren.

- Reaktionsgefäss in ein thermostatiertes Wasserbad von $40,0^{\circ} \mathrm{C}$ eintauchen und während 15 Minuten thermostatieren.

- In der Zwischenzeit Sistierlösungen vorbereiten (5 Messkölbchen).

- Die Sistierlösung enthält in jedem 50-ml-Messkölbchen je $30 \mathrm{ml}$ Sistier-Pufferlösung

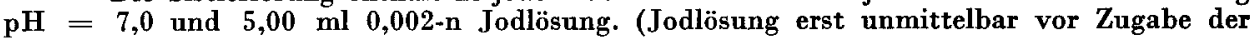
Reaktions-Lösung zusetzen).

- Lösungen im Reaktionsgefäss nach 15 Minuten langem Thermostatieren gut mischen und gleichzeitig eine Stoppuhr in Gang setzen (entspricht Reaktionszeit $=0$ ).

- In Zeitabständen von 2, 6, 12, 20 und 35 Minuten Proben von je 1 ml entnehmen (Oxford-Pipette), in vorher vorbereitete Sistierlösung einpipettieren und mit Wasser auf $50 \mathrm{ml}$ auffüllen.

- Messkölbchen nach gutem Durchmischen in ein Wasserbad von $20^{\circ} \mathrm{C}$ einstellen.

c) Blindlösung

— in 50 -ml-Messkölbchen abmessen :

- $30 \mathrm{ml}$ Pufferlösung $\mathrm{pH}=7,0$,

- 0,50 ml Honig-Lösung $2 \%$,

- 5,00 ml 0,002-n Jod, mit Wasser auf $50 \mathrm{ml}$ auffüllen.

d) Ausmessen

Die Extinktion der Blaufärbung wird sofort nach beendetem Enzymversuch, oder spä-

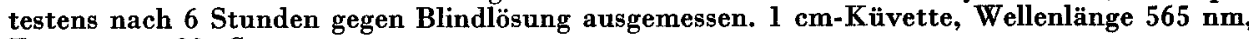
Temperatur $20^{\circ} \mathrm{C}$.

\section{Berechnung}

- Auf Millimeterpapier die gemessenen Extinktionswerte (Ordinate) gegen die Reaktionszeit (Abszisse) auftragen. Je nach Aktivität biegt die am Anfang linear verlaufende Kurve nach verschiedenen Zeiten ab. (Siehe Abb 2).

- Durch den linearen Teil der Kurve eine Regressionsgerade ziehen. ablesen.

- Im Schnittpunkt dieser Geraden mit der Ordinate den Anfangs-Extinktionswert E。 ablesen.

— Bei der Reaktionszeit 30 Minuten den Extinktionswert $\mathbf{E}_{\mathrm{t}}$ auf der Regressionsgeraden

— Diastasezahl (DZ) nach folgender Formel berechnen :

$$
\mathrm{DZ}=\frac{1500 \cdot\left(\mathrm{E}_{0}-\mathrm{E}_{\mathrm{t}}\right)}{\mathrm{E}_{0} \cdot t} .
$$

Es bedeuten $\mathbf{E}_{0}=$ Anfangs-Extinktion, graphisch ermittelt,

$\mathrm{E}_{\mathrm{t}}=$ Extinktion nach $t$ Minuten (graphisch ermittelt),

$t=$ Reaktionszeit (nach Vorschrift 30 Minuten).

\section{SACCHARASEZAHL}

Die Saccharasezahl wurde nach einer polarimetrischen Methode, welche ursprünglich von Duisberg und Gebelein (1958) stammte und von uns (HADoRN und ZứrCHER 1966) etwas modifiziert worden ist, bestimmt. Vergleiche auch Schweiz. Lebensmittelbuch (1967).

$$
\text { HYDROXYMETHYLFURFUROL (HMF) }
$$

Das Hydroxymethylfurfurol bestimmten wir nach WINKLER (1955).

$$
\text { TROCKENSUBSTANZ REFRAKTOMETRISCH }
$$

Siehe Schweiz. Lebensmittelbuch (1967). 
pH-WERT UND SAUREGEHALT

Diese Bestimmungen erfolgten potentiometrisch mittels Glaselektrode (HaDors und ZürCHER 1963).

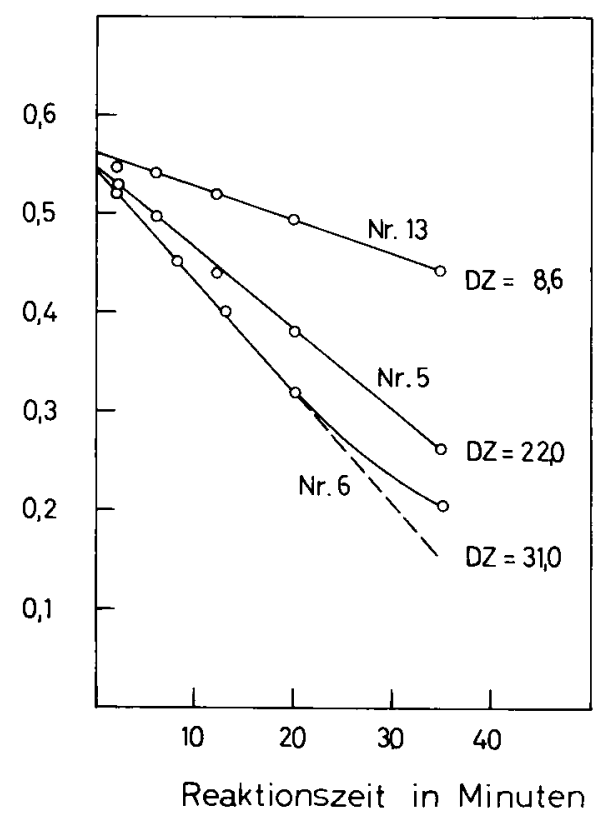

Aвв. 2. - Diastasezahl- Bestimmung; Reaktionskurve verschiedener Honige Nr. 6 Blïtenhonig Kanton Waadt.

Nr. 5 Blütenhonig Kanton Waadt

Nr. 13 Lavandin-Honig (Provence)

Fig. 2. - Détermination de l'indice de diastase; courbe de réaction pour diffêrents miels.

No 6 Miel de fleurs du canton de Vaud.

No 5 Miel de fleurs du canton de Vaud.

No 13 Miel de Lavandin de Provence.

En abscisses : temps de réaction en minutes

En ordonnées : extinction

\section{ERGEBNISSE}

Die Untersuchungsergebnisse sind in den Tabellen 1 bis 4 zusammengestellt und sollen kurz diskutiert werden.

\section{Reproduzierbarkeit}

Alle von uns benutzten Methoden liefern gut reproduzierbare Werte. Die kinetische Methode zur Bestimmung der Diastasezahl gibt bei Parallelbestimmungen gut übereinstimmende Werte. Bei 4 Analysen, welche an verschiedenen 
Tagen am gleichen Honig durchgeführt wurden, fanden wir Diastasezahlen welche zwischen 30,0 und 31,0 schwankten. Mittelwert $=30,4$; Standardabweichung $= \pm 0,5$. Der Wiederholstreubereich für eine statistische Sicherheit von $95 \%$ berechnet sich zu $\pm 1,5$ Einheiten.

\section{Einfluss des Stärkepräparates}

Im Handel findet man lösliche Stärkepräparate mit sehr unterschiedlichen Eigenschaften. Stärkepräparate, in denen die Stärke noch kaum abgebaut worden ist, geben auf Zusatz von Jodlösung eine Fällung von schwer löslicher Jödstärke. Derartige Präparate sind für die Diastasezahl-Bestimmung ungeeignet. Andere Stärkepräparate, beispielsweise lösliche Stärke nach ZuLKOWSKY, enthalten sehr stark abgebaute oder dextrinierte Stärke. Sie geben mit Jodlösung keine Blaufärbung sondern nur rötlich-braune Lösungen. Derartige Präparate sind ebenfalls ungeeignet. Als gut brauchbar erwiesen sich die Noredux-Standardstärke (Siegfried AG, Zofingen) und die lösliche Stärke von Merck. Wir haben Versuche mit 7 verschiedenen Stärkepräparaten des Handels durchgeführt und mit all diesen Stärken recht gut vergleichbare Diastasezahlen erhalten. (ZürCHER und HADORN 1972).

\section{Vergleich der kinetischen Methode mit anderen Methoden}

Wir haben unsere Methode auch mit der Gothe-Methode (1914) und der Methode Schade, Marsh und Eckert (1946) verglichen. In drei Honigen mit stark unterschiedlicher Diastaseaktivität bestimmten wir die Diastasezahl nach den drei Methoden. Die Resultate sind in der Tabelle 1 aufgeführt. Man erhält durchwegs vergleichbare Werte. Wie früher gezeigt wurde (HADoRN 1961), hängt die Diastasezahl nach GoTHE sehr stark von der Stärkequalität ab. Verwendet man die im Schweiz. Lebensmittelbuch vorgeschriebene Standardstärke, erhält man Werte, welche gut mit den nach unserer neuen kinetischen Methoden gefundenen übereinstimmen. Die Methode Schade, Marsh und EGKert (1958) lieferte durchwegs etwas niedrigere Resultate.

\section{Untersuchungen an authentischen Honigen}

Wir haben 11 Schweizerhonige und 7 französische Honige untersucht. Die Resultate sind in der Tabelle 2 zusammengefasst. Bei allen untersuchten Honigmustern handelt es sich um authentische, unter schonenden Bedingungen gewonnene Honige. Der Gehalt an Hydroxymethylfurfurol ist fast duchwegs niedrig $(0,1-0,5 \mathrm{mg} \%)$, woraus geschlossen werden darf, dass die Honige keiner übermässigen Wärmebehandlung unterworfen worden waren. Eine Ausnahme machen die französischen Honige Nr. 13 und 18, welche während 4. Tagen bei $30^{\circ} \mathrm{C}$ gelagert wurden (décanté 4 jours à $30^{\circ} \mathrm{C}$ ) und erst anschliessend kühl bei $14^{\circ} \mathrm{C}$ aufbewahrt wurden. Der Hydroxymethylfurfurol-Gehalt 
dieser beiden Honige ist durch die Wärmebehandlung deutlich erhöht worden. (1,23 bezw. 0,89 mg \%) Der französische Honig Nr. 12 wurde nach dem Schleudern während 14. Tagen bei $25^{\circ} \mathrm{C}$ aufbewahrt und erst anschliessend bei $14^{\circ} \mathrm{C}$ gelagert. Auch dieser Honig weist einen erhöhten HMF-Gehalt auf $(0,83 \mathrm{mg} \%)$.

TAB. 1. - Bestimmung der Diastasezahl nach verschiedenen Methoden

TABL. 1. - Détermination de l'indice de diastase selon différentes méthodes

\begin{tabular}{|c|c|c|c|}
\hline $\begin{array}{l}\text { Methode } \\
\text { Méthode }\end{array}$ & $\begin{array}{c}\text { No } 2 \\
\text { Kastanienhonig } \\
\text { Fontanaz-Seulaz } \\
\text { s. Bex }(800 \mathrm{~m}) \\
\\
\text { Miel de châtaignier } \\
\text { Fontanaz-Seulaz } \\
\text { s. Bex }(800 \mathrm{~m})\end{array}$ & $\begin{array}{l}\text { No } 13 \\
\text { Blütenhonig } \\
\text { 1971 von Crau } \\
\text { Südfrankreich } \\
\\
\text { Miel de fleurs } \\
\text { de Crau 1971 } \\
\text { (France) }\end{array}$ & $\begin{array}{c}\text { No } 14 \\
\text { Lavandin-Honig } \\
\text { Sept. 1971 } \\
\text { Valréas (Vaucluse) } \\
\\
\text { Miel de Lavandin } \\
\text { Valréas (Vaucluse) } \\
\text { Sept. 1971 }\end{array}$ \\
\hline Neue kinetische Methode & $\begin{array}{ll}30,0 & 30,3 \\
30,2 & 31,0\end{array}$ & $\begin{array}{ll}24,4 & 23,5 \\
22,3 & 23,9\end{array}$ & $\begin{array}{l}9,2 \\
8,6\end{array}$ \\
\hline Nouvelle méthode cinétique & & & \\
\hline $\begin{array}{l}\text { Methode Gothe, modifiziert nach Kiermeir u. } \\
\text { Köberlein } \\
\text { Méthode Gothe modifiée selon Kiermeier et Köberlein }\end{array}$ & 28,6 & 21,0 & 8,7 \\
\hline $\begin{array}{l}\text { Methode Schade, Marsh und Eckert, Modifika- } \\
\text { tion Hadorn LB } 23 \text { A /S. } 28 \\
\text { Méthode Schade, Marsh et Eckert, modifiée Hadorn LB } \\
23 \text { A /S.28 }\end{array}$ & 24,2 & 16,8 & 8,0 \\
\hline
\end{tabular}

Die Diastasezahlen der untersuchten Honige bewegen sich in normalen Grenzen, das heisst zwischen ca. 9 und 30 Einheiten. Besonders enzymreich, vor allem reich an Honigdiastase sind die beiden Kastanienhonige $\mathrm{Nr}$. 1 und Nr. 2. Auch die Saccharasezahlen der beiden Honige sind hoch. Auffallend niedrige Enzymaktivitäten besitzen die beiden Lavandin-Honige Nr. 14 und 15 aus Südfrankreich. Dies kommt besonders deutlich bei der Saccharaseaktivität zum Ausdruck. Diese beiden Lavandin-Honige besitzen Saccharasezahlen von 1,3 bezw. 3,2 ; dies sind die niedrigsten von uns je in einem authentischen Honig festgestellten Enzymaktivitäten. Die Diastasezahlen dieser Lavandin-Honige liegen mit 9, bezw. 11 Einheiten noch innerhalb der normalen Grenzen. Auf die natürliche Enzymarmut gewisser Honige werden wir noch zurückkommen.

Die weiteren Gehaltszahlen wie Wassergehalt, pH-Wert und Säuregehalt bewegen sich innerhalb normaler Grenzen und geben keinen Anlass zu besonderen Bemerkungen. 


\section{Enzymarme Honige}

Es ist seit einiger Zeit bekannt, dass gewisse authentische und unter schonenden Bedingungen gewonnene Honige von Natur aus relativ enzymarm sind. So fallen besonders Orangenblüten- und Lavendelhonige durch ihre Enzymarmut auf. Da die Honigenzyme zur Hauptsache aus dem Pharynxdrüsen-Sekret der Bienen stammen und dem Nektar während der Honigbereitung beigemischt werden, ist nicht ohne weiteres verständlich, weshalb gerade die Orangen- und Lavendelblüten-Honige besonders enzymarm sein sollten. Es wurde vermutet, dass diese Honige gewisse Stoffe enthalten könnten, welche als Enzymgift wirken und die Honigenzyme hemmen könnten. NeLson (1930) hat bereits darauf hingewiesen, dass als Träger des Aromas im Orangenblütenhonig das Methylanthranilat, ein Bestandteil des Orangenblütenöles, anzusehen ist. Er hat diese Verbindung aus Orangenhonig isoliert. Dieser Befund wurde von Lotrop (1932) bestätigt. Auch Deshusses und GabBaI (1962) haben in spanischen Orangen- und Lavendelhonigen mittels Dünnschicht-Chromatographie Methylanthranilat nachgewiesen. Da Methylanthranilat chemisch dem Vitamin $\mathbf{H}^{\prime}$, einigen wichtigen Lokalanästhetika (Anästhesin) und gewissen chemischen Konservierungsmitteln nahe verwandt ist, haben wir geprüft, ob Methylanthranilat im Honig enzymhemmend wirkt. Eigene Versuche (HAdorN 1964) haben ergeben, dass durch relativ hohe Zusätze von Methylanthranilat zu Honig (50 mg/100 g Honig) die Aktivität der Diastase überhaupt nicht, die der Honig-Saccharase nur minim gehemmt wird. Unter den uns zur Verfügung stehenden französischen Honigen befanden sich, wie bereits erwähnt, 2 Lavandin-Honige mit niedrigen Diastase- und Saccharasezahlen (DZ $=9,0$ und 11,$1 ; \mathrm{SaZ}=1,3$ und 3,2 ). Es sollte nun untersucht werden, ob in diesen enzymarmen Honigen irgend eine enzymhemmende Substanz vorkommt. Zu diesem Zweck haben wir den enzymarmen Honig mit einem enzymreichen Kastanienhonig im Verhältnis 1 : 1 gemischt und die Diastasezahl der Mischung bestimmt. Wenn eine enzymhemmende Substanz vorhanden wäre, müsste dieselbe auch die aus dem Kastanienhonig stammende Diastase hemmen. Die Mischung würde in diesem Fall eine niedrigere Diastasezahl besitzen als sich diese aufgrund der Mischungsrechnung aus den Diastaseaktivitäten der beiden Honige errechnen lässt. Aus den Resultaten der Tabelle 3 geht hervor, dass keine Enzymhemmung stattfindet. Das Vorhandensein irgend eines enzymhemmenden Stoffes kann mit grosser Wahrscheinlichkeit ausgeschlossen werden. Die Tatsache, dass in der Natur öfters auffallend enzymarme Honige vorkommen, bleibt jedoch bestehen. Es wäre auch möglich, dass gewisse, im Nektar von Orangen- und Lavandin-Blüten enthaltene Stoffe, wie Methylanthranilat, auf die Sekretabsonderung oder auf die Enzymproduktion der Pharynx-Drüsen der Bienen hemmend wirken, so dass von Anfang an ein enzymarmer Honig zubereitet wird. Gegen diese Theorie 
H. HADORN, K. ZÜRCHER

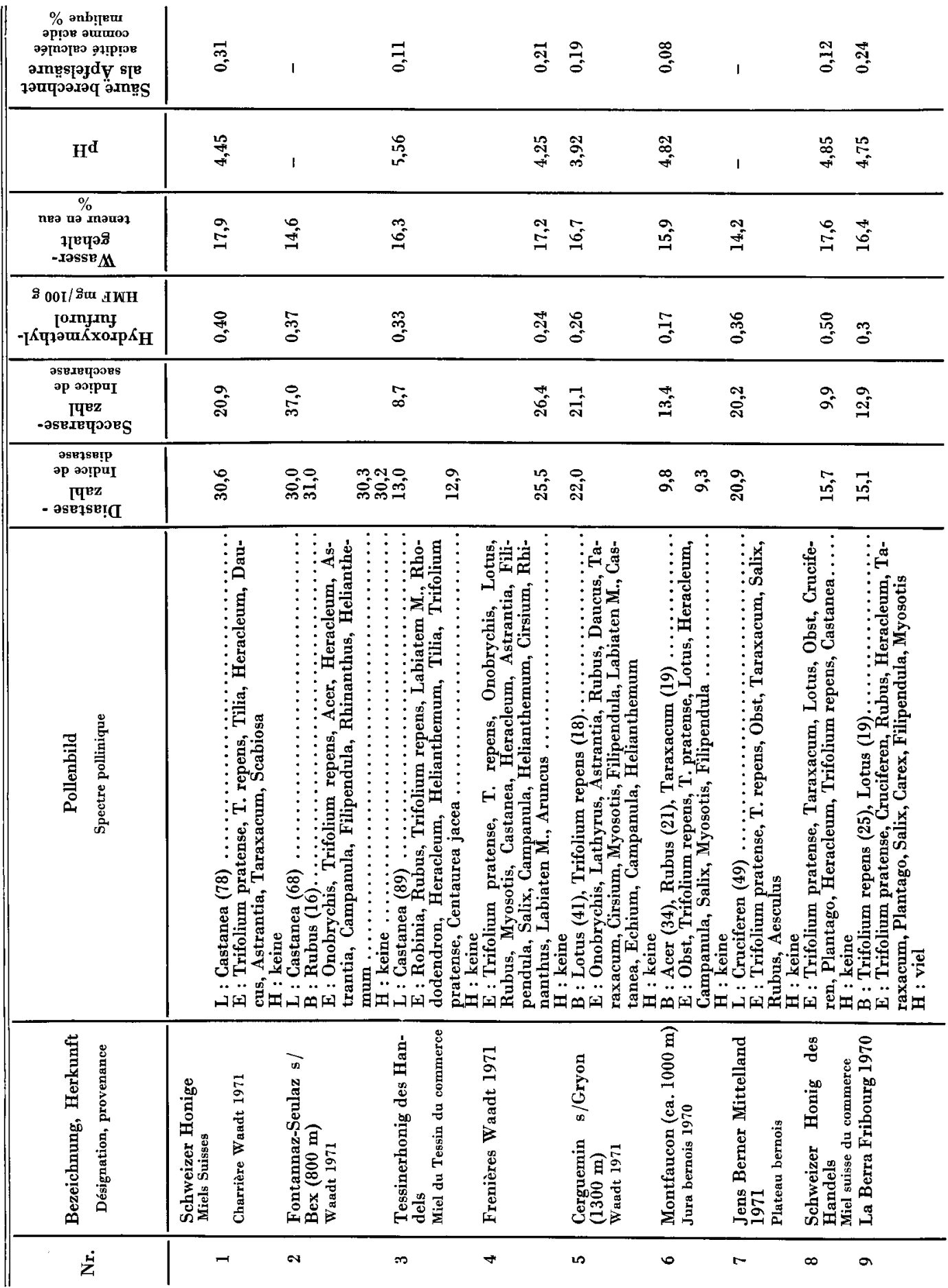




\begin{tabular}{|c|c|c|c|c|c|c|c|c|}
\hline ֻั & $\stackrel{-1}{8}$ & $\vec{m}_{0}^{\vec{m}}$ & 1 & 1 & 1 & 1 & 1 & $\stackrel{\text { 영 }}{\circ}$ \\
\hline$\underset{+}{8}$ & $\begin{array}{l}\text { 㤐 } \\
\text { in }\end{array}$ & $\stackrel{\sim}{F}$ & 1 & 1 & 1 & 1 & 1 & $\underset{\sim}{\mathbb{N}}$ \\
\hline 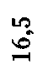 & : & 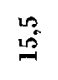 & 弪 & 今̊ & $\vec{f}$ & $\vec{\triangleq}$ & $\vec{s}$ & $\stackrel{m}{\stackrel{0}{0}}$ \\
\hline$\approx$ & $\stackrel{9}{\circ}$ & 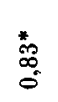 & 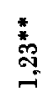 & : & 융 & $\begin{array}{l}\infty \\
\stackrel{\infty}{0} \\
0\end{array}$ & 囱 & 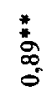 \\
\hline ک & $\stackrel{8}{=}$ & 茴 & $a^{2}$ & $=$ & 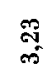 & $\stackrel{\infty}{\sim}$ & $\tilde{\infty}$ & $\stackrel{\infty}{\sim}$ \\
\hline
\end{tabular}

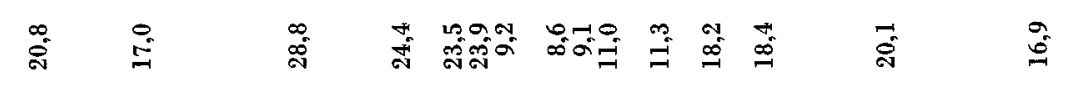

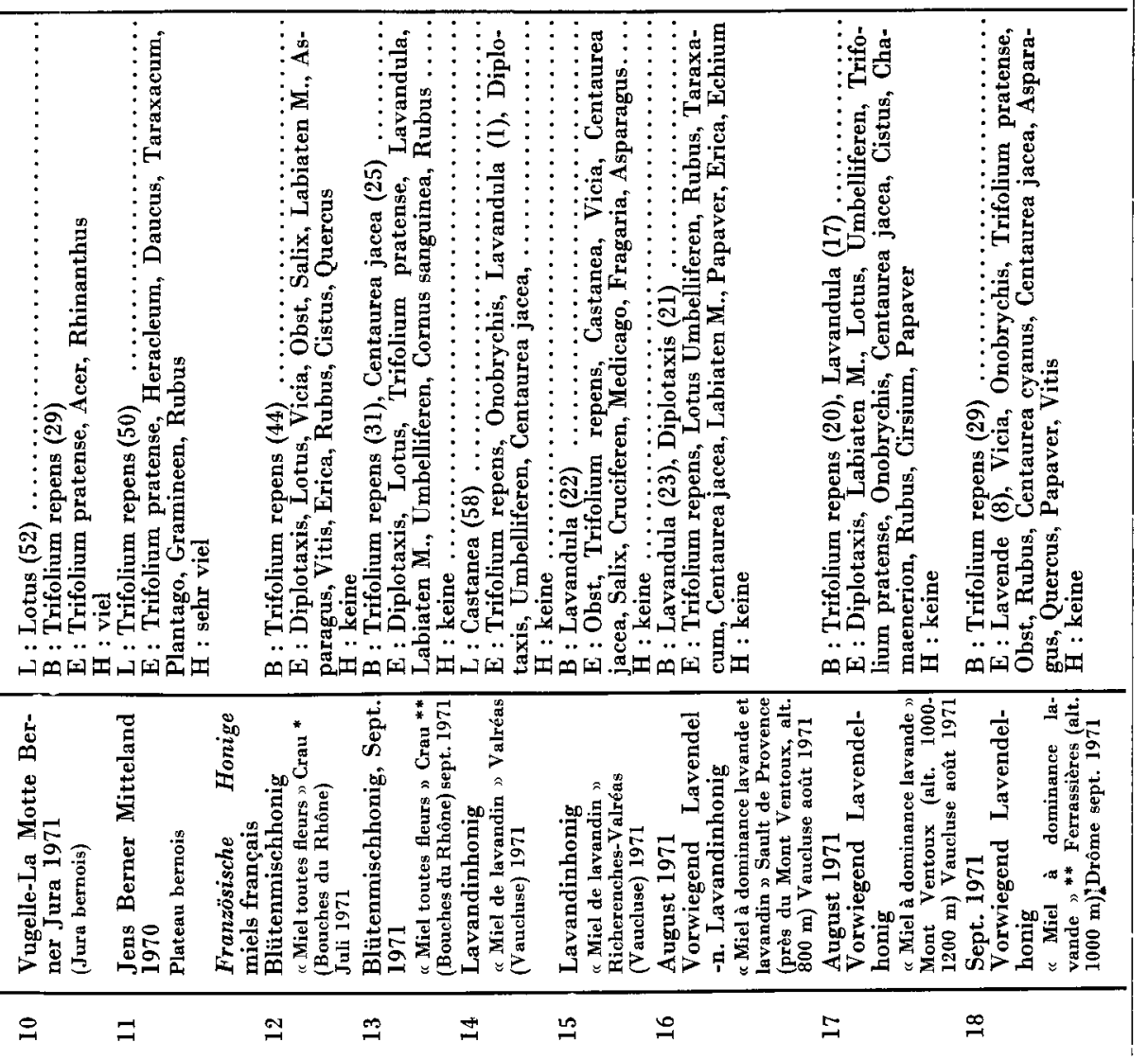

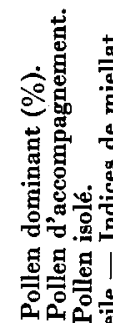
कर० $111 \%$ के s응 . क || || || $\|$ 山田的国

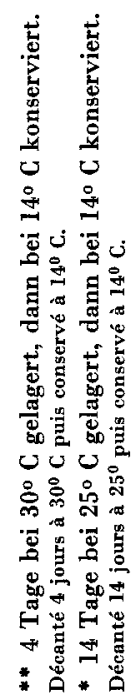


spricht jedoch der Umstand, dass nicht ausnahmslos alle Lavandin- und Orangenblüten-Honige enzymarm sind. Fermentarmut des Honigs kann unter bestimmten Bedingungen, etwa bei ausgesprochener Massentracht und gleichzeitigem Mangel an Pollen, auftreten. Lavandin-Tracht in der Provence ist meistens Massentracht, da einzelne Völker pro Tag oft über $5 \mathrm{~kg}$ Nektar eintragen.

Taв. 3. - Versuche zur Prüfung auf Enzymhemmung

TABL. 3. - Essais de blocage des enzymes

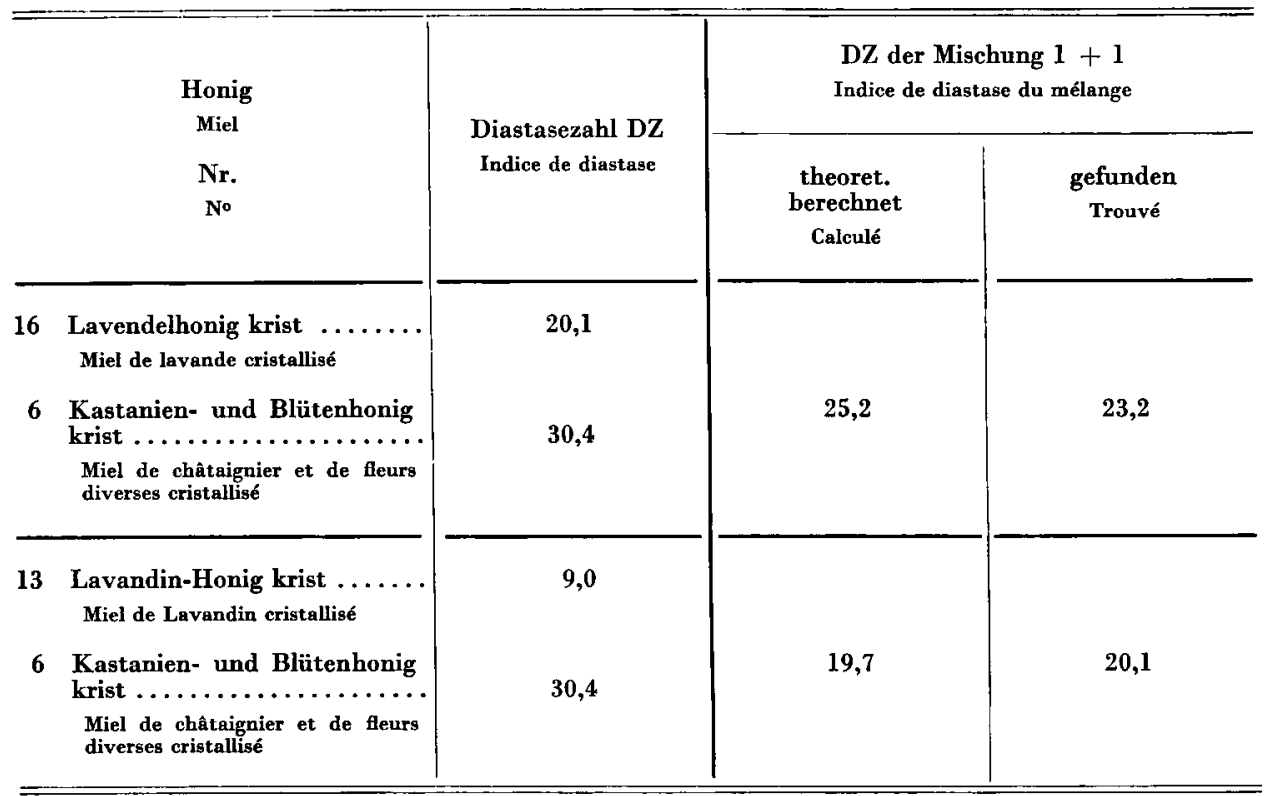

\section{Herkunft und Eigenschaften der Honig-Diastase}

Ueber die Herkunft der Honigenzyme liegt eine Anzahl Untersuchungen vor. Nach der heutigen Auffassung stammen die Honigenzyme (Diastase, Saccharase, Glukose-Oxydase) zur Hauptsache aus den Sekreten der im Kopf und Thorax der Biene gelegenen Drüsen, die während des Reifungsprozesses dem Honig beigemengt werden. Die wichtigste Rolle spielt dabei das an Enzymen reiche Sekret der Pharynxdrüsen (Maurizio et al. (1972), Oerosi (1968), Ziegler (1968)). Gewisse Mengen von Saccharasen sind auch in den Rohstoffen selbst, vor allem im Honigtau, nachgewiesen worden (KLoFT (1960), (1968), Kloft /Maurizio/Käser (1965), Maurizio (1965), (1968a, b).

Um indirekt einen Hinweis auf die Herkunft der Honigdiastase zu erhalten, haben wir gewisse Eigenschaften der Honigdiastase studiert. Pflanzliche und tierische Amylase sollen unterschiedliche Eigenschaften aufweisen. So 
schreibt beispielsweise Street (1962) auf Seite 854 wörtlich : « Tierische Amylase wird durch Chlorid-Ionen aktiviert. Maximale Aktivität erhält man bei dem hier beschriebenen Verfahren, wenn die Chlorid-Konzentration im Ansatz der enzymatischen Reaktion grösser als 0,01-n ist. Ein Chloridgehalt bis zu $0,9 \%$ stört nicht. Pflanzliche Amylase wird durch Chlorid-Ionen nicht aktiviert $)$.

TAB. 4. - Aktivierung der Honigdiastase durch Natriumchlorid

TABL. 4. - Activation de la diastase du miel par le chlorure de sodium

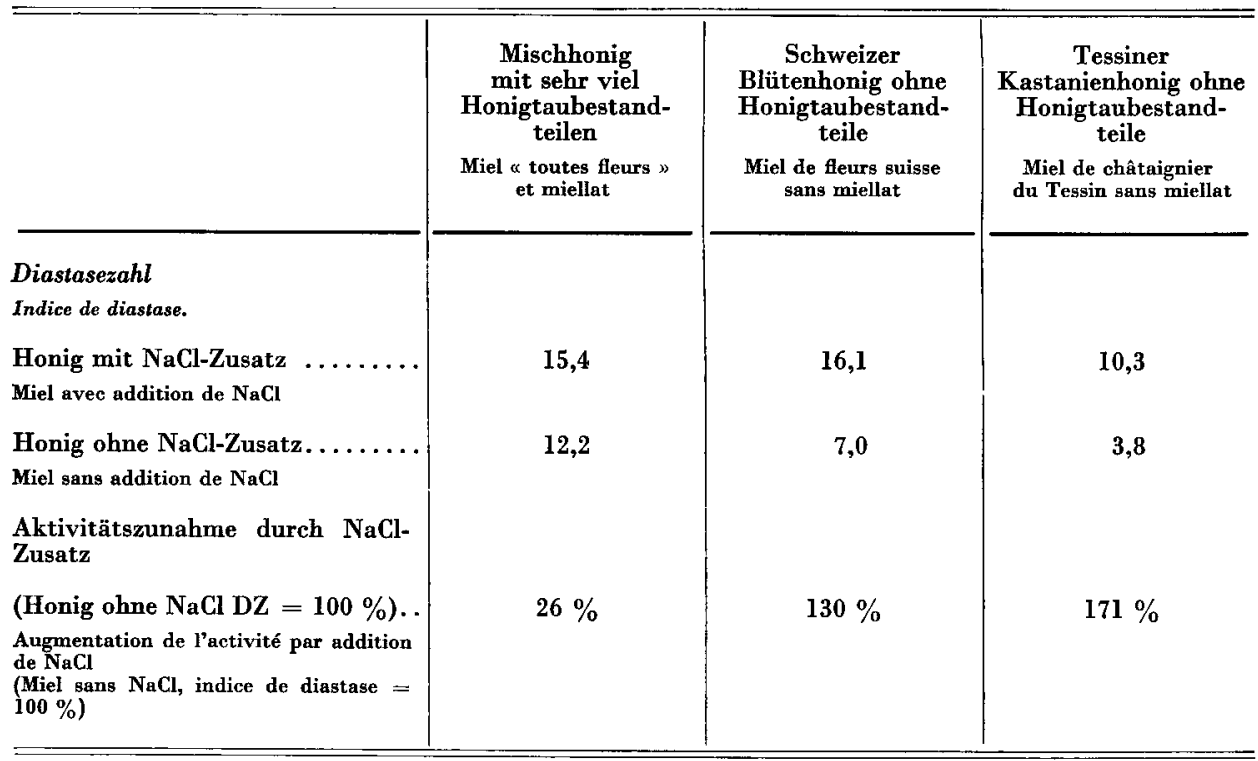

Wir haben in einem Honigtau-Honig und in zwei Blütenhonigen die Aktivierung der Amylase durch Natriumchlorid studiert. Die Resultate sind in der Tabelle 4 aufgeführt. Durch den Natriumchlorid-Zusatz wird die Aktivität der Honigdiastase in allen Honigen deutlich erhöht. Beim reinen Blütenhonig und beim Kastanienhonig ohne Honigtau-Bestandteile steigt die Aktivität auf den 2 bis nahezu den dreifachen Anfangswert. Beim Mischhonig mit viel Honigtau-Bestandteilen ist die Aktivitätserhöhung durch Natriumchlorid geringer. Möglicherweise hängt dies damit zusammen, dass Honigtau-Honige von Natur aus bereits einen höheren Mineralstoff-Gehalt aufweisen. Die beobachtete Aktivierung der Honigdiastase durch Natriumchlorid dürfte beweisen, dass die in obigen Honigen enthaltene Diastase vorwiegend tierischer Herkunft ist. Zwischen Honigtauhonig und Blütenhonig besteht kein prinzipieller Unterschied.

Zur weiteren Charakterisierung der Honigdiastase der drei Honige wurde das pH-Optimum ermittelt. Zu diesem Zweck haben wir die Diastase-Aktivität 
nach der angegebenen Methodik ermittelt, jedoch in verschieden gepufferten Lösungen. Aus Abb. 3 geht deutlich hervor, dass das pH-Optimum der Diastase

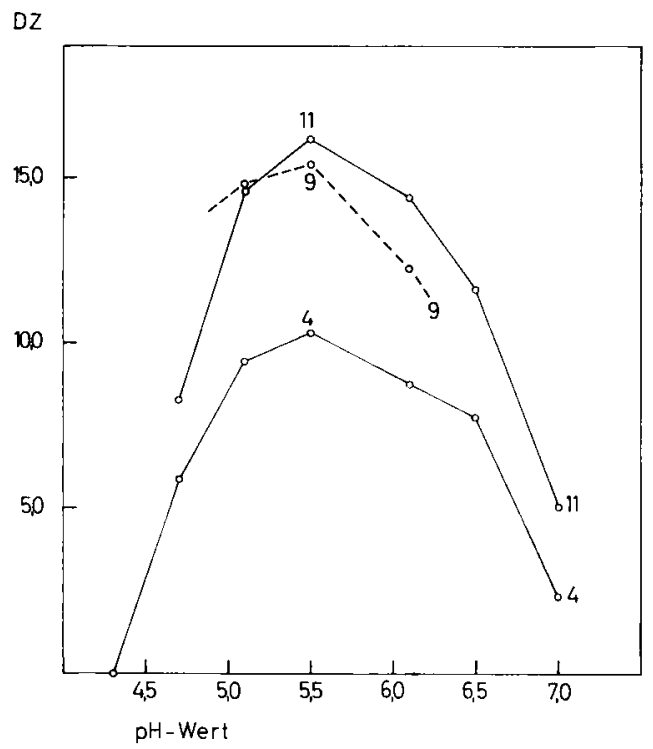

Aвв. 3. - - pH-Optimum der Honig-Diastase

Nr 4 Tessiner Kastanienhonig, keine Honigtaubestandteile Nr. 9 Mischhonig aus dem Berner Mittelland, viel Honigtaubestandteile

Nr. 11 Schweizer Bienenhonig, keine Honigtaubestandteile

FIG. 3. - - pH optimum de la diastase du miel

No 4 Miel de châtaignier du Tessin, pas de miellat

No 9 Miel toutes fleurs du pays bernois, beaucoup de miellat

No 11 Miel suisse, pas de miellat

En abscisses : pH

En ordonnées : indice de diastase

bei allen drei Honigen bei $\mathrm{pH}=5,4$ liegt. Bei früheren Versuchen (HAdORN 1961) fanden wir in Kastanienhonig das pH-Optimum der Diastaseaktivität zwischen $\mathrm{pH}=5,1$ und $\mathrm{pH}=5,6$, was gut mit obigen Befunden übereinstimmt. Aufgrund dieser Versuche enthalten Blütenhonige und Honigtau-Honige anscheinend die gleiche Diastase. Dieselbe ist tierischer Herkunft, sie stammt zum grössten Teil aus den Pharynxdrüsen der Bienen, zum Teil möglicherweise auch aus dem Honigtau, welcher eine Ausscheidung von Blattläusen darstellt. Die Honigdiastase unterscheidet sich deutlich von der menschlichen Serum-Amylase (a-Amylase), welche nach STrEeT ein Aktivitätsoptimum bei pH $=7,0$ besitzt.

Eingegangen im April 1972.

Reçu pour publication en avril 1972. 


\section{DANK}

Frl. Dr. Anna Maurizio, Liebefeld-Bern, hat uns eine Anzahl authentischer SchweizerHonige überlassen und die Pollenanalyse durchgeführt. Wir möchten ihr an dieser Stelle für ihre wertvolle Mitarbeit, vor allem für Literaturhinweise und die Durchsicht unserer Arbeit, bestens danken.

Herr Dr. J. Louveaux, Bures-sur-Yvette, Frankreich, hat uns einige Honige aus Südfrankreich zugestellt und Angaben über die Pollenanalyse gemacht. Auch ihm danken wir für seine Bemühungen bestens.

\section{RÉSUME}

Une méthode cinétique pour la détermination de l'indice de diastase du miel a été mise au point. La méthode repose sur le principe suivant : une solution mère tamponnée de miel réagit à $40^{\circ} \mathrm{C}$ sur une solution d'amidon. $A$ intervalles de temps déterminés on prélève des échantillons du mélange en cours de réaction et on y ajoute une solution d'iode. On suit la dégradation de l'amidon par voie photométrique. Sur un diagramme on porte respectivement la valeur d'extinction et le temps de réaction correspondant. On calcule l'indice de diastase à partir de la courbe de réaction qui, au début, est linéaire.

La nouvelle méthode donne des valeurs reproductibles. Les résultats sont en accord avec ceux que l'on obtient avec la méthode de Goxhe. Par rapport à la méthode de Schade, Marsi et ECKerT (modification dans le recueil des méthodes utilisées en Suisse pour l'analyse des produits alimentaires) on obtient des valeurs qui sont un peu plus basses.

La méthode a été essayée sur 11 échantillons de miels suisses d'origine connue ainsi que sur 7 échantillons de miels français. A côté de l'indice de diastase nous avons déterminé pour ces miels l'indice de saccharase, la teneur en H.M.F. et quelques autres valeurs intéressantes. Parmi les miels analysés qui sont tous d'origine bien définie on a trouvé quelques miels particulièrement riches en enzymes (indice de diastase allant jusque 31 ; indice de saccharase allant jusque 37) mais, également des miels d'une pauvreté en enzymes surprenante. Deux miels français de lavandin venant de Provence ont donné des indices de saccharase extrêmement faibles (respectivement 1,3 et 3,2). Les indices de diastase de ces deux miels variaient respectivement entre 9 et 11 , donc à l'intérieur des limites normales. Dans les miels pauvres en enzymes nous n'avons pu mettre en évidence la présence d'aucune substance inhibitrice. Si l'on mélange un miel pauvre en diastase (Lavandin) avec un miel riche en diastase (Châtaignier) dans la proportion de $1: 1$, on trouve dans le mélange exactement l'activité diastasique qui peut être calculée de façon théorique. La pauvreté naturelle en enzymes de certains miels devrait être en rapport avec une miellée massive.

Une addition de chlorure de sodium active l'activité diastasique aussi bien du miel de fleurs que du miellat. Ceci semble indiquer que la diastase du miel est d'origine animale. En effet, les amylases d'origine végétale ne sont pas activées par le chlorure de sodium. Pour poursuivre la caractérisation de la diastase du miel nous avons déterminé chez plusieurs miels le pH optimum. Celui-ci est de 5,4 aussi bien pour les miels de fleurs que pour les miellats. Selon nos expériences les miels de fleurs et les miellats contiennent une diastase qui a les mêmes propriétés. La teneur en hydroxyméthylfurfurol des miels d'origine connue et non altérés par la chaleur est faible. Elle se situe entre 0,1 et $0,5 \mathrm{mg} / 100 \mathrm{~g}$. Lorsqu'il y a conservation du miel pendant plusieurs jours à $25-30^{\circ} \mathrm{C}$ la teneur en H.M.F. augmente sensiblement. 
H. HADORN, K. ZÜRCHER

\section{IITERATUR}

Deshusses J., Gabbai A., 1962, Recherche de l'anthranilate de méthyle dans les miels espagnols de fleur d'oranger par chromatographie sur couche mince. Mitt. Lebensmitteluntersuch- und Hyg. (Bern) 53, 408-411.

Duisberc H., Gebeleiv H., 1958, Ueber die Kontrolle von Erhitzungsschäden bei Honigen. ZUL 107, 489-503.

Gothe F., 1914, Experimentelle Studien über Eigenschaften und Wirkungsweise der HonigDiastase. ZUL, 28, 286-321.

Hadorn H., 1961, Zur Problematik der quantitativen Diastasebestimmung in Honig. Mitt. Lebensmitteluntersuch. und Hyg. (Bern) 52, 67-103.

Hadorn H., Zürcher K., 1963, Formolzahl von Honig; gleichzeitige Bestimmung von Formolzahl, pH, freier Säure und Lactongehalt in Honig. Mitt. Lebensmitteluntersuch. und Hyg. (Bern) 54, 304-330.

HadoRs H., 1964, Enthalten Orangenblüten- und Lavendelblüten- Honige enzymhemmende Stoffe? Annales de l'abeille 7, 311-320.

HAdoRN H., ZürCHER K., 1966, Eine verbesserte polarimetrische Methode zur SaccharasezahlBestimmung in Honig. Dtsch. Lebensmitt.-Rundschau 62, 195-201.

Kiermeier F., Köberleiv W., 1954, Ueber die Hitzeinaktivierung von Enzymen in Honig. ZUL 98, 329-347.

KLOFT W., 1960, Wechselwirkungen zwischen p flanzensaugenden Insekten und den von ihnen besogenen Pflanzengeweben. Z. ang. Entom. 45, 337-381; 46, 42-70.

Kloft W., Maurizio A., Kaeser W., 1965, Das Waldhonigbuch, Ehrenwirth Verlag München.

KLofT W., 1968, Les insectes producteurs de miellat. Traité de Biologie de l'Abeille Vol. 3, 248-263 Masson, Paris.

Lotrop R.-E., 1932, Ein spezifischer Nachweis von Orangen-Honig. Ind. a Eng. Chem. Analyt. Ed. 4, 395-396. (Referat in Z. Lebensmittelunters. u. Forsch. 1937, 73, 585).

Maurizio A., 1965, Honigentstehung-Honigreifung. D. Bienenwirtsch. 16, 233-240.

Maurizio A. 1968 a, b, La formation du miel; Les diastases des glandes nourricières. Traité de Biologie de l'Abeille Vol. 3, 264-276; Vol. 1, 291-301, Masson, Paris.

Maurizio und Mitarbeiter. Der Honig. Ulmer Verlag Stuttgart. (im Druck).

Nelsov E.-K., 1930, Das Aroma des Orangen-Honigs. Ind. Engin. Chem. 22, 448. (Referat in Z. Lebensmittelunters. u. Forsch. 1936, 72, 588).

Oerösi-Pal Z., 1968, Physiologie des glandes nourricières. Traité de Biologie de l'Abeille. Vol. 1, 263-290, Masson Paris.

Schade J.-E., Marsh G.-L., Eckert J.-E., 1958, Diastase Activity and Hydroxy-MethylFurfural in Honey and their Usefulness in Detecting Heat Alteration. Food Research 23, 446-463.

Schweizerisches Lebensmittelbuch, 1967, Kapitel 23, Honig und Kunsthonig. Eidg. Drucksachen- und Materialzentrale Bern.

Street H.-V., 1962, Methoden der enzymatischen Analyse. Herausgegeben von H.-U. BergMEYER, Verlag Chemie, Weinheim.

White J.-W., Pairent F.-W., 1959, Report on the Analysis of Honey. J. Assoc. Offic Agr. Chemists (AOAC) 42, 341-348.

Winkser O., 1955, Beitrag zum Nachweis und zur Bestimmung von Oxymethylfurfurol in Honig und Kunsthonig. ZUL 102, 161-167.

Ziegler H., 1958, La sécrétion du nectar. Traité de Biologie de l'Abeille. Vol. 3, 218-248, Masson Paris.

Zürcher K., Hadorn H., 1972, Eine einfache kinetische Methode zur Bestimmung der Diastasezahl in Honig. Dtsch. Lebensmitt.-Rundschau 68, 209-216. 